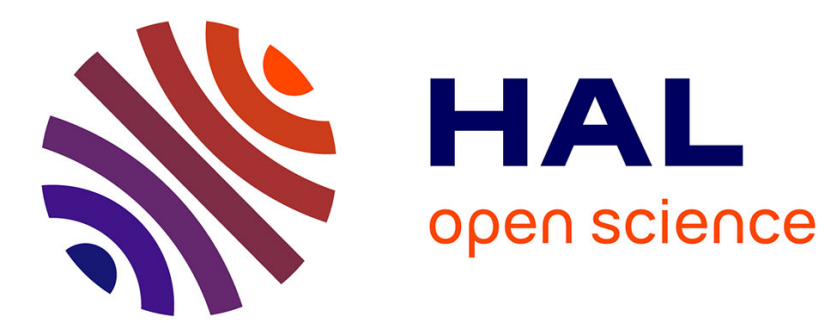

\title{
An Endorectal Ultrasound Probe Comanipulator With Hybrid Actuation Combining Brakes and Motors
}

\author{
Cécile Poquet, Pierre Mozer, Guillaume Morel, Marie-Aude Vitrani
}

\section{To cite this version:}

Cécile Poquet, Pierre Mozer, Guillaume Morel, Marie-Aude Vitrani. An Endorectal Ultrasound Probe Comanipulator With Hybrid Actuation Combining Brakes and Motors. IEEE/ASME Transactions on Mechatronics, 2015, 20 (1), pp.186-196. 10.1109/TMECH.2014.2314859 . hal-01170908

\section{HAL Id: hal-01170908 \\ https://hal.science/hal-01170908}

Submitted on 10 Jul 2015

HAL is a multi-disciplinary open access archive for the deposit and dissemination of scientific research documents, whether they are published or not. The documents may come from teaching and research institutions in France or abroad, or from public or private research centers.
L'archive ouverte pluridisciplinaire HAL, est destinée au dépôt et à la diffusion de documents scientifiques de niveau recherche, publiés ou non, émanant des établissements d'enseignement et de recherche français ou étrangers, des laboratoires publics ou privés. 


\title{
An underactuated comanipulator for safely positioning a needle in a prostate under endorectal ultrasound guidance
}

\author{
Cécile Poquet, Pierre Mozer, Guillaume Morel, Marie-Aude Vitrani
}

\begin{abstract}
A robotic device, aimed at assisting a urologist who positions an endorectal ultrasound probe to perform prostate biopsies, is presented.

The proposed system is a comanipulator that holds the probe simultaneously with the urologist. This robot supports two modes of operation: the free mode, where the entire movement control is left to the urologist when he/she positions the probe with respect to the prostate thanks to the feedback provided by the ultrasound images; the locked mode, where the robot's role is to precisely maintain the targeted biopsy site at a given location, while the urologist can insert a needle through a guide mounted to the probe and proceed to biopsy.

The device combines three brakes and three motors. This allows both transparent comanipulation in the free mode with 6 Degrees of Freedom (DoFs) liberated and stabilization of the probe in the locked mode. At the control level, a main challenge in the locked mode raises from antagonistic constraints: the needle placement shall be precise in spite of unknown external forces due to the contact between the probe and the rectum; the robot apparent impedance shall be low due to security constraints. This is solved by an inner low stiffness controller and an outer slow integration for canceling steady state errors.

Both in vitro and in cadavero experimental results show the efficiency of the system in the two modes of operation.
\end{abstract}

\section{INTRODUCTION}

In 2013, more than 230.000 new prostate cancer cases have been detected in the USA, thanks to hundreds of thousands biopsy procedures [1]. Prostate biopsy is indeed the medical examination used to diagnose a prostate cancer. It consists of sampling the prostate tissue using a biopsy needle.

An examination shall include twelve samples equally distributed across the prostate volume. A major technical difficulty arises from the desired precision for the needle placement, which is typically a few $\mathrm{mm}$ [2], [3]. Achieving such a precision is difficult because the prostate has a variable volume, experiences large displacements (up to $1 \mathrm{~cm}$ ), and significantly deforms [4], [5]. Meanwhile, obtaining a high precision and control of the 3D needle placement constitute a major medical issue for the prostate biopsy procedure. Indeed, it may lead to obtaining a fine 3D map of cancerous regions in the prostate which is required for allowing the development of local therapy instead of total prostate ablation, which is today the most common treatment of prostate cancer. Prostatectomy

Authors are with UPMC Univ. Paris 06, UMR 7222, ISIR, Paris, France \{poquet, mozer, morel, vitrani\}@isir.upmc.fr.

P. Mozer is also from La Pitié Salpetrière Hospital, Urology Dpt., France

Part of this work has already been published in the IRSJ/IEEE Int. Conf on Robots and Systems, Oct. 2013 induces a high rate of side effects, such as incontinence and is more and more considered as an unnecessary surgery for a number of patients, accounting for the very slow development of certain cancers. The development of local therapy (namely by creating a necrose in a small region of the prostate) will be possible only when the biopsy procedure precision will be significantly increased as compared to current practice. Note also that placing a needle in a prostate with a high precision is required for brachytherapy, which consists of inserting radioactive seeds through a needle across the prostate volume in order to irradiate the cancerous tissue.

Because of its crucial importance in terms of public health, robotic assistance to needle placement in the prostate has been the object of interest for the robotics community in the past years. A recent exhaustive overview of these systems can be found in [6].

Imagery is a first feature that can be used to classify the systems proposed across the literature. Because the prostate deforms and moves during a needle placement procedure, it is required to monitor the needle placement by intraoperative imaging. Across the literature, authors propose to use ultrasound imaging (USI) [6]-[8], MRI [9]-[11] or CT Scan [12]. USI provides either 2D planar images in real time or 3D images at a few seconds rate. 2D USI is often coupled with a stepper: thanks to successive incremental penetration movements of the probe, a series of parallel cross-sections are acquired and assembled to provide a 3D image [7], [8], [13]-[16]. USI is largely available in the urologist cabinet for a reasonable cost. MRI or CT scan imaging provide better images at a higher cost and a lower frequency. MRI also imposes drastic constraints on the design of the robot due to magnetic compatibility [9], [11] and CT scan brings problems due to irradiation doses for both the urologist and the patient. In order to be compatible with the medico-economic constraints of the biopsy procedure and in accordance with the most common practice across urologists worldwide, our system, called Apollo, exploits endorectal ultrasound imaging.

Robotic systems found in the literature can also be classified by the needle access: needles can be placed in the prostate either through a transperineal access, [6], [12]-[18], or through a transrectal access, [19], [20]. In the clinical conventional practice, for a biopsy procedure, a transrectal access is exclusively adopted. The patient is placed in a lateral decubitus position and a local anesthesia of the rectal region is performed. He is awake and thus he may move during the procedure. Endorectal needle placement is usually associated 
with endorectal ultrasound imaging: the needle guide is fixed with respect to the USI probe and both are inserted simultaneously in the rectum. As a result, the needle position is known in the probe image frame. It can be visualized by a straight line in the screen displaying the image, prior to its insertion. Transperineal needle placement is generally used for brachytherapy. Here, the patient is placed in the lithotomy position. Some authors also suggest to use transperineal access for biopsies [11], but the procedure is slower and the requirement of a total anesthesia seems not compatible with the medico-economic constraints. Apollo exploits a transrectal access for the needle, through a guide attached to the USI probe, because it is compatible with the current practice and simplifies the robot design without adding constraints due to imagery.

The robot kinematics is the third factor distinguishing the existing robots that assist the placement of a needle in the prostate. The number of Degrees of Freedom (DoFs) required to place the tip of a needle at an arbitrary position with an arbitrary needle axis orientation is 5 and not 6 , since the rotation of the needle around its penetration axis does not affect the tip position nor the axis orientation. Some authors use 6 active DoFs, the actuation of the rotation around the needle axis being used to improve the needle penetration through the perineum, [6]. For robots manipulating an endorectal USI probe, like Apollo, the anus plays the role of a 2 DoFs kinematic constraints. Only 4 DoFs are to be used: 3 rotations around the penetration point and 1 translation along the penetration axis. This has led to the design of robots exhibiting a remote center of motion [14]. A clear benefit of this approach is that only 4 actuated degrees of freedom are required, which participates to reducing the cost. A major drawback is that, prior to operation, a setup phase is required to put the remote center, which is fixed with respect to the robot base, and the patient's anus in coincidence. Moreover, in a study where the endorectal USI probe displacements during clinical practice have been monitored, it has been observed that the anatomical constraint is not perfectly respected during manual operation [21]. Due to other geometrical constraints (from anatomy and from needle guide placement that should leave an easy access to the urologist), it seems to be useful to produce small movements that do not strictly leave the entry point at a fixed position. For these reasons, Apollo possesses six degrees of freedom in such a way that its placement with respect to the patient's anatomy is not imposed by a remote center constraint, and the urologist can slightly displace the anus when required for an optimal probe placement.

Finally, the last criteria for classifying the literature is the degree of automation. Some devices are fully automated: the robot is registered with respect to the prostate, the needle desired localization is given by a pre-operative planning and the robot places autonomously the needle [15]. This is of particular interest for devices guided by CT Scan images, since the urologist can stand far away from the CT Scanner, reducing the exposure to irradiations. Some devices are comanipulators, in that sense that the gesture control is shared by the robot and the urologist. Most frequently, with this approach, the robot positions the needle guide while the needle placement, by itself, is done by the urologist [8], [14], [19]. Apollo fits in the category of comanipulators, although it differs from the existing systems by the functions is provides. Instead of separating between robotic autonomous probe placement and human needle placement, it leaves the urologist positioning the probe.

Our observation of the clinical practice is that the probe placement is a complex task, during which the urologist monitors the real time US images while accounting for a number of constraints, such as: maintaining a sufficient pressure to the rectal wall in order to properly image the prostate; limiting the pressure to the rectal wall in order to avoid large deformation or displacement of the prostate; respecting the fulcrum point constraint - to a certain extent; controlling the rotation of the probe around its penetration axis to obtain both an adequate needle target position and a reasonable access to the needle guide from its proximal placement. Regarding this last point, it is worth noticing that the biopsy target is a $3 \mathrm{D}$ point in the prostate. Meanwhile, constraints on orientation are loose. In other words, for a given target, the urologist can chose different probe orientations, particularly for the orientation of the probe around the penetration axis. The rules used by the urologist to solve this task kinematic redundancy seem to be adapted to a number of intraoperative parameters and not easily reproducible by an autonomous robot. To our knowledge, this redundancy question has not been fully addressed in the literature so far.

All in one, realizing such a complex movement with a robotic device would require a precise knowledge of all the patient's anatomical constraints and does not leave the freedom to adapt to intraoperative clinical unpredictable events, such as prostate displacements or even patient's movements. This is why, with our system, which is described in detail in Sect. IV, the probe positioning is left to the urologist. During this clinical phase, the robot, whose end-effector is attached to the probe, shall leave the probe motion as free as possible. This first function is called the free mode. It is presented and evaluated through in vitro experiments in Sect. III. Once the probe has been manually positioned, Apollo shall provide a second function: the locked mode, during which the urologist has his/her hands free to perform the needle placement and the biopsy. Here, it is desired that the robot maintains precisely the target position, while preserving the patient's safety. This is antagonistic in the context of robot control: usually precision is achieved thanks to a high stiffness while safety, for a robot in contact with a human, requires a low impedance. The locked mode is presented, together with in vitro experiments, in Sect. IV. Finally, two cadavers experiments are reported, confirming the performances observed during in vitro experiments, see Sect. V.

\section{Proposed System}

\section{A. Apollo's kinematics}

A picture of Apollo is given in Fig. 1. As justified in Sect. I, it exhibits 6 DOFs to be compatible with all the required probe motion while avoiding to constrain its placement with respect to the patient. While the robot base is placed at an approximate 


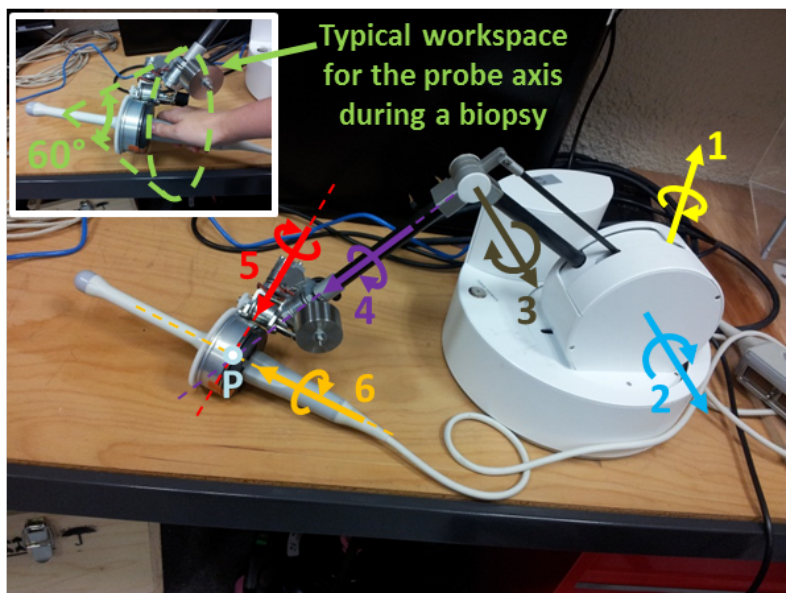

Figure 1. A general view of the proposed TRUS probe comanipulator

distance of $40 \mathrm{~cm}$ from the entry point, on the examination table, it allows the probe to cover the required workspace. This workspace was determined from clinical data in [21], during a prostate biopsy procedure. It can be modeled by a cone, whose origin coincides with the anatomical entry point, and the angle is typically 60 degrees, see Fig. 1, upper left corner. Apollo is made of 6 pivot joints serially assembled according to a conventional anthropomorphic geometry: the 3 first joints form the shoulder and the elbow, while the wrist is composed of the three last joints, whose axes coincide at a point $P$, see Fig. 1 . The geometry is completely described by Table I according to Denavit and Hartenberg notation, [22].

\begin{tabular}{||c|c|c|c|c||}
\hline$i$ & $\alpha_{i-1}$ & $a_{i-1}$ & $d_{i}$ & $\theta_{i}$ \\
\hline 1 & 0 & 0 & 0 & $\theta_{1}$ \\
\hline 2 & $-\pi / 2$ & 0 & 0 & $\theta_{2}$ \\
\hline 3 & 0 & $0.25 \mathrm{~m}$ & 0 & $\theta_{3}$ \\
\hline 4 & $\pi / 2$ & 0 & $0.3 \mathrm{~m}$ & $\theta_{4}$ \\
\hline 5 & $-\pi / 4$ & 0 & 0 & $\theta_{5}$ \\
\hline 6 & $\pi / 2$ & 0 & 0 & $\theta_{6}$ \\
\hline \multicolumn{5}{|c|}{ Table I }
\end{tabular}

DENAVIT AND HARTENBERG PARAMETERS OF THE COMANIPULATOR

The last pivot axis is designed in such a way that it leaves a $8 \mathrm{~cm}$ diameter cylindrical hole whose axis coincides with the rotational axis. Therefore, an interface part can be designed to adapt to any specific probe shape and to connect to the robot end-effector. This part is fixed on the probe and can be placed into the robot end effector thanks to a mechanical connector involving magnets, see Fig. 2

\section{B. Actuation}

In order to obtain the locked mode, the system must be actuated. Since there is no need for active motion, a first guess solution is to mount brakes on all the 6 robot's joints. However, this would require an infinite stiffness for both the brakes and the robot structure. Indeed, once the urologist has positioned the probe at a desired location and sets up the locked mode, he/she releases the probe handle to manipulate the needle and the biopsied tissues. Then, all the external forces that the urologist was compensating for in the free mode, namely

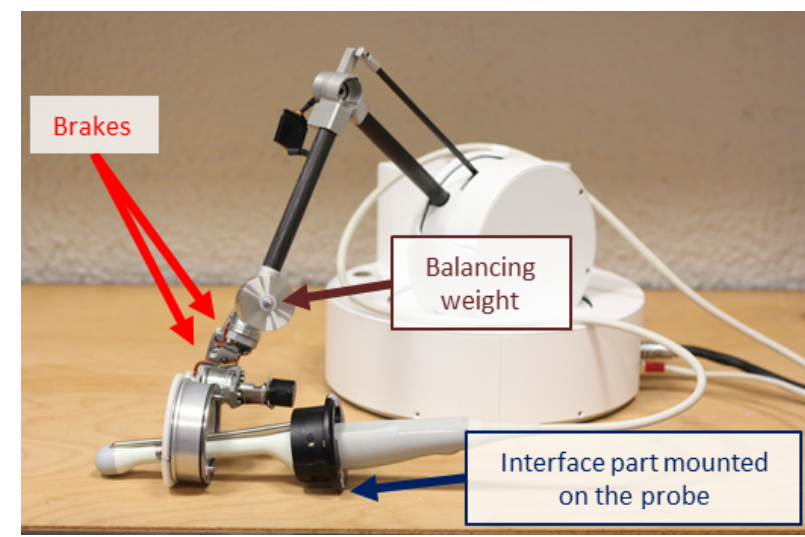

Figure 2. A view of the interface part used to mount the probe. It allows a 340 degrees rotation of the probe around its axis. A hole is left to insert the biopsy needle guide.

the probe weight and the interaction wrench applied to the patient through the probe, act as disturbances for the robot when he/she releases the probe. If the robot's stiffness with the brakes on is not infinite, this will lead to a displacement of the targeted site. Achieving a very high stiffness for both the robot structure and the brakes can be done only by increasing the robot weight and the brakes power. All together, this would be detrimental to the robot's lightness (or transparency), which is crucial for the free mode.

In order to maintain a high transparency (low friction, low weight, low inertia) for the robot's free mode, while being able of maintaining the biopsy target at a precise location despite unknown disturbances in the locked mode, a hybrid actuation system iss chosen:

- For the three wrist joints, small electromagnetic brakes are installed (Kebco 01.P1.300). The control of the brakes is binary: the brakes are either blocked $(\mathrm{ON})$, which corresponds to the unpowered state, or free (OFF), which corresponds to powered state. Therefore, in case of a loose of power, the wrist will be freezed to its configuration. Brakes provide a null torque when they are OFF. When they are ON, they exhibit a high resistive torque with a low mass. Note that because the stiffness is not infinite, a displacement of the probe under the disturbing external forces may be observed, resulting in a displacement of the biopsy target point $T$. This displacement can be measured thanks to high resolution potentiometers mounted on the joints' axes.

- To be able to compensate for the possible displacements due to external forces, electric motors (Maxon RE35) are mounted on the three first joints. In order to maintain a high transparency in the free mode:

- the motors are placed near the robot base, in such a way that their mass does not significantly affect the robot's inertia.

- a cable transmission is used to limit the joint friction.

- load springs are mounted on joints 2 and 3 to compensate for the robot weight.

Each motor is equipped with an optical encoder for position measurement. In the low level electronics, a 
current loop allows controlling the motor torque. The control input for the three first joints' motors is the current $i_{i}, i \in\{1 \cdots 3\}$, which corresponds to a joint torque $\tau_{i}$ up to a scalar factor $k_{\tau_{i}}$ accounting for the motor torque constant and the transmission ratio:

$$
\tau_{i}=k_{\tau_{i}} i_{i}, \quad i \in\{1 \cdots 3\} .
$$

In the following, the torque is considered as the control input for the three first joints' motors, knowing that the corresponding input current can be computed thanks to Eq. (1).

The robot has been fabricated by the French company Haption, [23], and exploits the Haption technology dedicated to high forces haptic interfaces for the three first joints. All the characteristics of the actuation system are summarized in Table II.

\begin{tabular}{||c|c|c|c|c||}
\hline Joint & Actuator & Transmission & Ratio & Max. torque \\
\hline 1 & motor & cable & 21.6 & $3.4 \mathrm{Nm}$ \\
\hline 2 & motor & cable & 14.9 & $2.4 \mathrm{Nm}$ \\
\hline 3 & motor & cable & 14.8 & $2.3 \mathrm{Nm}$ \\
\hline 4 & brake & direct & 1 & $0.4 \mathrm{Nm}$ \\
\hline 5 & brake & direct & 1 & $0.4 \mathrm{Nm}$ \\
\hline 6 & brake & gear & 4.5 & $1.8 \mathrm{Nm}$ \\
\hline
\end{tabular}

Table II

ACTUATION DATA

\section{FREE MODE}

\section{A. Control}

The computation of the torques for the free mode is primarily based on the kinematic model:

$$
\left(\begin{array}{c}
\mathbf{v}_{6 / 0}(P) \\
\omega_{6 / 0}
\end{array}\right)=\underbrace{\left(\begin{array}{cc}
\mathbf{J}_{v 1, P} & \mathbf{0} \\
\mathbf{J}_{\omega 1} & \mathbf{J}_{\omega 2}
\end{array}\right)}_{\mathbf{J}} \dot{\mathbf{q}},
$$

where $\mathbf{v}_{a / b}(M)$ stands for the velocity of point $M$ produced in the motion of frame $\mathscr{F}_{a}$ with respect to frame $\mathscr{F}_{b}, \omega_{a / b}$ stands for the rotational velocity of frame $\mathscr{F}_{a}$ with respect to frame $\mathscr{F}_{b}, \dot{\mathbf{q}}=\left[\begin{array}{lll}\dot{\theta}_{1} & \cdots & \dot{\theta}_{6}\end{array}\right]^{\mathrm{T}}$ is the joint velocity vector, $\mathbf{J}$ is the $6 \times 6$ robot jacobian matrix at point $P$ and $\mathbf{J}_{v 1, P}, \mathbf{J}_{\omega 1}$ and $\mathbf{J}_{\omega 2}$ are $3 \times 3$ jacobian sub-matrices. Note that the upper right null sub-matrix indicates that the three last joint movements do not affect the velocity of point $P$, which is the points where the wrist axes intersect.

In the rest of the paper, we will assume full rank for $\mathbf{J}$ (and thus for $\mathbf{J}_{v 1, P}, \mathbf{J}_{\omega 1}$ and $\mathbf{J}_{\omega 2}$ ), which is practically guaranteed in the prototype due to joint physical limits that leave kinematic singularities out of the workspace.

Due to kinemato-static duality, the transpose of the jacobian matrix defined in Eq. (2) can be used to map an external wrench applied to the environment through the end-effector into the vector of joint torques $\tau=\left[\begin{array}{lll}\tau_{1} & \cdots & \tau_{6}\end{array}\right]^{\mathrm{T}}$ :

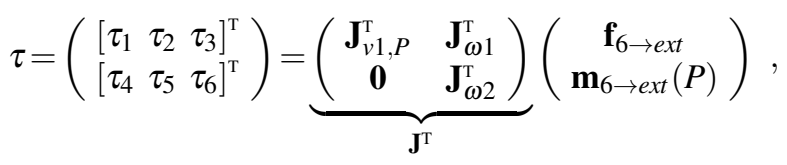

where $\mathbf{f}_{6 \rightarrow \text { ext }}$ is the force applied by the robot end-effector on the environment and $\mathbf{m}_{6 \rightarrow \text { ext }}(P)$ is the moment applied by the robot end-effector on the environment at point $P$. In the free mode, the brakes being OFF, the joint torques $\tau_{4}$ to $\tau_{6}$ are null. Therefore, from the second line of Eq. (3), it can be seen that the exerted wrench has a null moment at point $P$ : $\mathbf{m}_{6 \rightarrow \text { ext }}(P)=\mathbf{0}$. In other words, Eq. (3) simplifies to:

$$
\left(\begin{array}{lll}
\tau_{1} & \tau_{2} & \tau_{3}
\end{array}\right)^{T}=\mathbf{J}_{v 1, P}^{\mathrm{T}} \mathbf{f}_{6 \rightarrow e x t} .
$$

The robot links weight is balanced by counterweights and springs in such a way that there is no need for compensation of the robot weight by the actuators. However, to ease the comanipulation in the free mode and to cancel weight disturbances in the locked mode, it is desirable to compensate for the weight of the probe and its interface. The external wrench applied to the probe and its interface at point $P$, due to gravity is:

$$
\left(\begin{array}{c}
\mathbf{f}_{g \rightarrow 6} \\
\mathbf{m}_{g \rightarrow 6}(P)
\end{array}\right)=\left(\begin{array}{c}
m \mathbf{g} \\
m \mathbf{d}_{G P} \times \mathbf{g}
\end{array}\right),
$$

where $m$ and $G$ are the mass and the center of gravity of the probe and its interface, $\mathbf{g}$ is the gravitational field vector and $\mathbf{d}_{G P}$ is the vector from $G$ to $P$. Balancing experiments lead to identify $m=0.5 \mathrm{~kg}$ and $\mathbf{d}_{P G}=-d \mathbf{z}_{6}$, where $d=9 \mathrm{~mm}$ and $\mathbf{z}_{6}$ is the unit vector parallel to the probe penetration axis, directed towards the prostate.

Compensating for gravity in the free mode straightforwardly derives from Eq. (4) and Eq. (5):

$$
\tau_{g, f}=\left(\begin{array}{lll}
\tau_{1} & \tau_{2} & \tau_{3}
\end{array}\right)^{T}=-m \mathbf{J}_{v 1, P}^{\mathrm{T}} \mathbf{g} .
$$

This controller is referred in the next as FMC (Free Mode Control) and is depicted in Fig. 3.

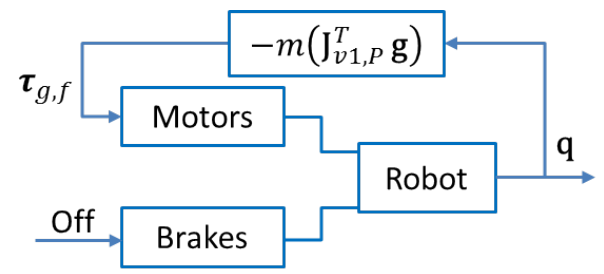

Figure 3. Free mode control scheme.

\section{B. Experimental evaluation}

Apollo's transparency was evaluated during in vitro experiments consisting of repeated pointing tasks with the probe passing through a hole. Fig. 4 shows the experimental setup. This simple task exhibits a probe kinematics and worspace that is comparable to those of a real biopsy task.

The probe distal part has been equipped with three laser beams which do not present any geometrical particularity (no parallel, no crossing point) as can be seen on Fig. 5. When the beams are on, they project three dots on a computer screen. As the probe passes through a hole which is attached to the table, one unique position and orientation of the probe goes with one unique placement of the three red dots on the screen (which is also fixed to the table). 


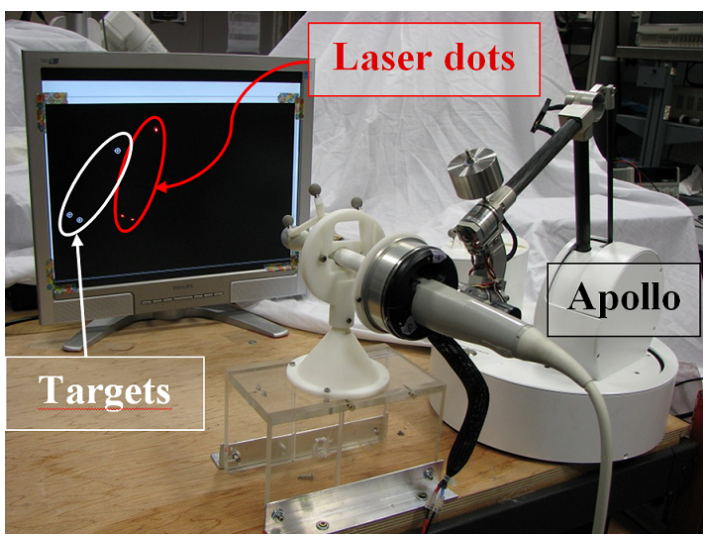

Figure 4. Experimental set-up to evaluate Apollo's transparency

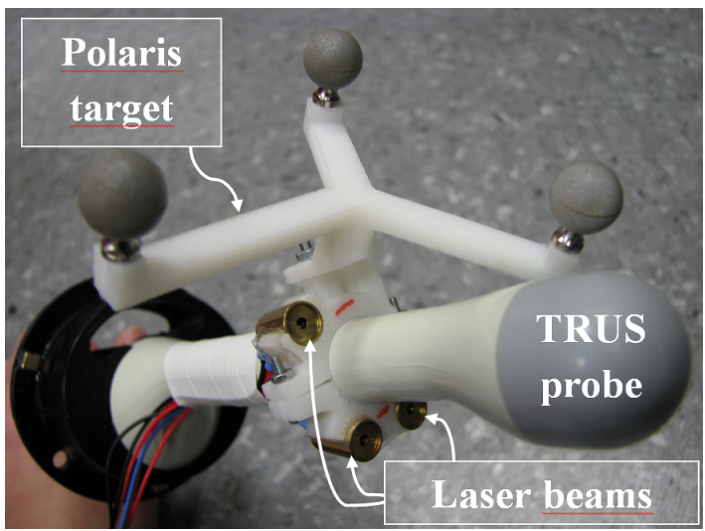

Figure 5. Distal part of the THRUS probe equipped with laser beams and a Polaris target

The subjects has been asked to perform pointing tasks. They were presented with an image made of three white targets on a black background, see Fig. 4. Once they had managed to place each of the laser dots in its target, an other image (thus an other set of targets) was displayed. A set of six images has been used repetedly.

In order to avoid any learning effect, the subjects were asked to perform the pointing task endlessly. Once the time needed to perform the task on one set of six images was stable, the task was considered learnt and the experiment itself began.

The subjects had to perform the pointing task six times on the set of images under each of the following conditions:

- without Apollo: holding the probe with their hand, the probe not being mounted on the robot,

- with Apollo: holding the probe with their hand, the probe being mounted on the robot and the robot compensating for gravity has presented before.

These two conditions were presented to each subjects in a random order.

To monitor the probe position wether it was mounted on Apolloor not, we used a Polaris which is a stereoscopic localization system. Two Polaris targets were used : one was fixed to the probe distal part, as can be seen on Fig. 5, the other was attached to the robot bas to be used as a reference.

\section{Results}

Twelve naive subjects have been involved in this experiment. Table III presents the average time in seconds used to perform the task on one set of images for each subject and condition, along with the global average time. The other metrics presented in this table is the average Spectral Arc Length (SAL) of the subjects movements which is dimensionless and represents the movements smoothness as explained in [24].

\begin{tabular}{|c|c|c|c|c|}
\hline \multirow[b]{2}{*}{ Subject } & \multicolumn{2}{|c|}{$\begin{array}{c}\text { Average time } \\
\text { by image set (in s) }\end{array}$} & \multicolumn{2}{|c|}{$\begin{array}{l}\text { Average SAL } \\
\text { by image set }\end{array}$} \\
\hline & $\begin{array}{l}\text { Without } \\
\text { Apollo }\end{array}$ & $\begin{array}{l}\text { With Apollo } \\
\text { in FMC }\end{array}$ & $\begin{array}{l}\text { Without } \\
\text { Apollo }\end{array}$ & $\begin{array}{l}\text { With Apollo } \\
\text { in FMC }\end{array}$ \\
\hline Subject 1 & 16.2 & 27.5 & -9.96 & -11.54 \\
\hline Subject 2 & 19.4 & 23.3 & -10.02 & -11.08 \\
\hline Subject 3 & 26.0 & 24.0 & -12.60 & -13.94 \\
\hline Subject 4 & 22.6 & 19.8 & -10.96 & -9.28 \\
\hline Subject 5 & 30.9 & 29.6 & -13.53 & -11.85 \\
\hline Subject 6 & 25.8 & 26.9 & -9.63 & -9.62 \\
\hline Subject 7 & 25.4 & 25.9 & -12.97 & -11.07 \\
\hline Subject 8 & 24.2 & 25.7 & -14.42 & -12.77 \\
\hline Subject 9 & 17.1 & 21.0 & -9.37 & -8.11 \\
\hline Subject 10 & 21.5 & 21.4 & -10.97 & -10.88 \\
\hline Subject 11 & 25.2 & 25.7 & -9.80 & -9.80 \\
\hline Subject 12 & 27.5 & 29.6 & -12.42 & -11.53 \\
\hline Average & 23.5 & 25.0 & -11.39 & -10.96 \\
\hline
\end{tabular}

SUMMARY OF THE DATA EXTRACTED DURING POINTING TASKS

Two student tests were performed on the full set of data (six values by subject and by condition, thus two groups of 72 values for each test) to asses the effect of Apolloon the task completion time and on the movements smoothness during a pointing task. We obtained p-values of respectively 0.0690 and 0.0796 .

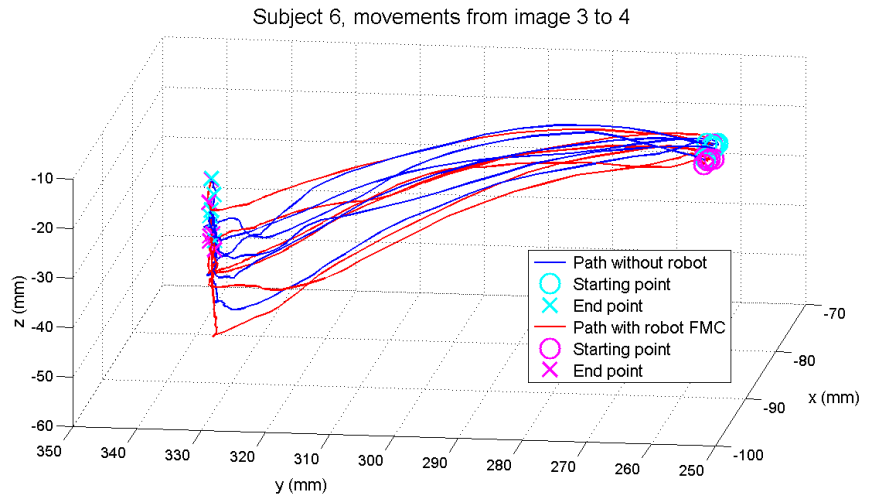

Figure 6. Movements of subject n6 between images 3 and 4, with and without Apollo

Fig. 6 shows some example trajectories. They were acquired on one of the subject when he was pointing image 4 starting from the image 3 pointing position.

\section{Discussion}

When one look at the full set of data summerized in Table III, it appears that the time needed to perform the 
pointing task on one set of images does not vary greatly with the robot presence. The same can be said of the SAL. As both p-values are under 0.05, the impact of Apollo's presence on those aspects of the task cannot be considered significant. Involving more subjects un the study might lead to statistically significant differences between the presence and the absence of Apollo. But those differences appear to be small enough to be acceptable:

- the completion time of the task is $1.5 \mathrm{~s}$ higher with the robot in free mode than without, which represents less than $7 \%$ of the average value; this would lead to a very limited increase in the biopsy examination;

- the SAL differs only by 0.43 which represents less than $4 \%$ of the average value; on Fig. 6 we can see indeed that movements performed with or without the robot cannot be easily differentiated. Furthermore, the mouvement is slightly smoother (e.g. the SAL value is closer to zero) when Apollois holding the probe and performing gravity compensation. Should the difference in SAL be significant, it would be on the advantage of our system.

Thus in free mode Apollo's presence as very little impact on a simple manipulation task that mimics the movements performed during prostate biopsies. The transparency can be considered satisfying for the considered application.

\section{LOCKED MODE}

\section{A. Control}

For the locked mode, we propose three different controllers, to be evaluated experimentally. Our aim is to evaluate whether the use of active brakes and/or position measurement is required for the wrist joints.

1) First controller: The first controller, hereinafter BMCA, does not use the brakes or the wrist joint position sensors. Our idea here is to use only the three motors to guarantee that, once the robot is switched to the locked mode, the position of point $P$ is maintained constant. In this case, because of the friction between the probe and the rectum, it may be possible to obtain a constant position and orientation. This will depend on the magnitude of the elastic forces between the probe and the patient, that may influence the probe orientation around point $P$. For this controller, the gravity compensation is the same as for the free mode, while a torque is added to emulate an elastic behavior at point $P$ :

$$
\tau_{B M C-A}=\tau_{g, f}+\tau_{k, P}
$$

where:

$$
\tau_{k, P}=\mathbf{J}_{v 1, P}^{\mathrm{T}} k\left(\mathbf{x}_{\mathrm{ref}, P}-\mathbf{x}_{P}\right) .
$$

In this last equation, $k$ is a stiffness coefficient, $\mathbf{x}_{P}$ is the position of point $P$ in the fixed frame $\mathscr{F}_{0}$, which can straightforwardly be computed from the three first joints position though the robot's direct kinematics model and $\mathbf{x}_{\mathrm{ref}, P}$ is the position of point $P$ recorded when the urologist activates the locked mode from the free mode. In other words, it is the position where point $P$ shall stay still.

It is desirable to tune a low stiffness for security reasons. Indeed, during a biopsy procedure, the patient may be moving and the resulting forces should not be too large. In practice, a stiffness as low as $k=200 \mathrm{~N} / \mathrm{m}$ is selected. As a result, it was experimentally found that the residual joint friction of the device, although rather low, was enough to damp out the oscillations without using a velocity feedback. However, in this case, the efforts applied to the rectum and the prostate may induce significant displacements for point $P$. To compensate for this, an outer integral compensation is added. The reference position is changed with a rate:

$$
\dot{\mathbf{x}}_{\mathrm{ref}, P}=\lambda\left(\mathbf{x}_{\mathrm{ref}_{0}, P}-\mathbf{x}_{P}\right),
$$

where $\lambda$ is a scalar gain in $s^{-1}$ and $\mathbf{x}_{\text {ref }_{0}, P}=\mathbf{x}_{\mathrm{ref}, P}$ when the urologists sets the locked mode on. In other words:

$$
\mathbf{x}_{\mathrm{re}, P}=\mathbf{x}_{\mathrm{ref}_{0}, P}+\lambda \int_{0}^{t}\left(\mathbf{x}_{\mathrm{ref}_{0}, P}-\mathbf{x}_{P}(\tau)\right) d \tau .
$$

Thanks to this integrator, when the urologist releases the probe after setting on the locked mode, the probe initially moves due to the wrench applied to the patient, but is compensated for thanks to a modification of the reference position.

Combining Eq. (10) with Eq. (7) and Eq. (8), one finally gets a controller in the locked mode that could be written as an equivalent conventional PI compensator for the position error, see Fig. 7. What is particular here is the external loop implementation for the integrator and the associated tuning method: a low stiffness $k$ is first chosen $(200 \mathrm{~N} / \mathrm{m})$; then, the external integral gain $\lambda$ is chosen to adjust the time required to compensate for a disturbance. It is not required to select a high value for $\lambda$. A slow compensation will ensure a correction of the position within a few seconds, which is acceptable for the clinical application. It will not significantly change the stiffness at the frequencies that are typical for a human-robot interaction (from 0.5 to $3 \mathrm{~Hz}$ ). Furthermore, for safety reasons, the integration can be stopped either when the error will have become null, after a few seconds, or when the force applied by the controller exceeds a tunable limit. In practice, the external integrator was tuned thanks to experiments in which an error of $1 \mathrm{~cm}$ induced by an external load should be corrected in approximately 5 second thanks to integration. This lead to $\lambda=4 \mathrm{~s}^{-1}$.

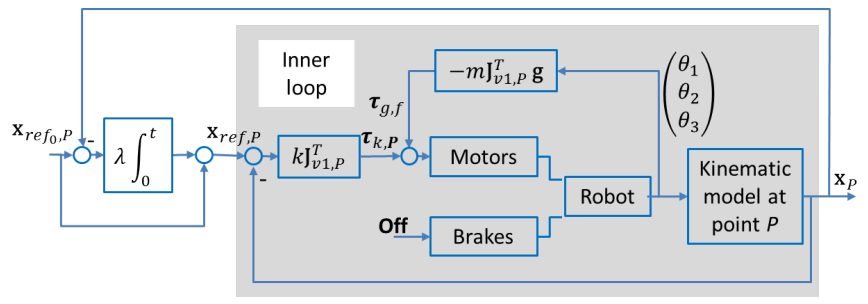

Figure 7. First control scheme in the locked mode (BMC-A)

2) Second controller: Because maintaining constant the position of point $P$ while the wrist is free may be insufficient to guarantee that the position of the biopsy target $T$ is fixed, a second controller is proposed that uses brakes and simultaneously emulates a spring behavior for point $P$. Note that, as long as the brakes are ON and do not slip, bodies 3, 4, 5 and 6 of the robot constitute a same solid body. The velocity 
transmission model is obtained from Eq. (2) when considering that the three last joint velocities are null:

$$
\left(\begin{array}{c}
\mathbf{v}_{6 / 0}(P) \\
\omega_{6 / 0}
\end{array}\right)=\left(\begin{array}{c}
\mathbf{J}_{v 1, P} \\
\mathbf{J}_{\omega 1}
\end{array}\right)\left(\begin{array}{c}
\dot{\theta}_{1} \\
\dot{\theta}_{2} \\
\dot{\theta}_{3}
\end{array}\right),
$$

Reciprocally, both a force and a moment can be applied to the probe at point $P$, but only three actuators are controlled. The mapping from an external wrench applied to the probe to the three active joints torques is obtained by the dual of Eq. (11):

$$
\left(\begin{array}{lll}
\tau_{1} & \tau_{2} & \tau_{3}
\end{array}\right)^{T}=\mathbf{J}_{v 1, P}^{\mathrm{T}} \mathbf{f}_{6 \rightarrow \text { ext }}+\mathbf{J}_{\omega 1}^{\mathrm{T}} \mathbf{m}_{6 \rightarrow \text { ext }}(P) .
$$

Therefore, the gravity compensation term is now, from a combination of Eq. (12) and Eq. (5):

$$
\tau_{g, b}=-m\left(\mathbf{J}_{v 1, P}^{\mathrm{T}} \mathbf{g}+\mathbf{J}_{\omega 1}^{\mathrm{T}}\left(\mathbf{g} \times \mathbf{d}_{G P}\right)\right)
$$

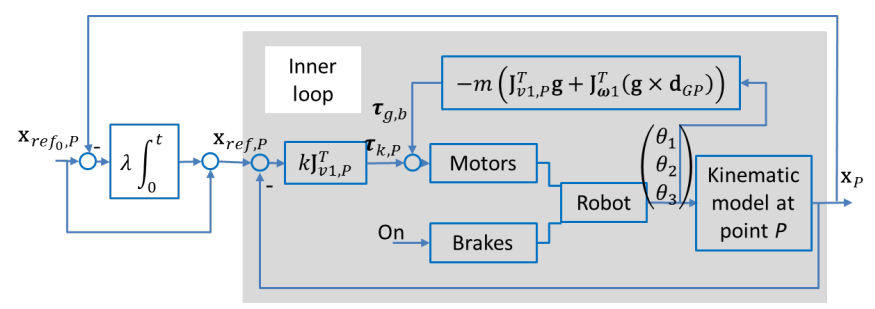

Figure 8. Second control scheme in the locked mode (BMC-B)

The second controller uses this new gravity compensation term in addition to the spring emulation at point $P$ :

$$
\tau_{B M C-B}=\tau_{g, b}+\tau_{k, P},
$$

where $\tau_{k, P}$ is defined in Eq. (8). Here again, in order to guarantee a good static precision for the positionning of point $P$, an external integrator is added, which leads to the controller depicted in Fig. 8. The same gain $\lambda=4 \mathrm{~s}^{-1}$ is used for the external intergator.

3) Third controller: Although brakes are used with the BMC-B controller, and although the external integrator ensures a null steady state error at point $P$, the system may suffer from a lack of positioning precision at point $T$. Indeed, the stiffness of the wrist brakes is not infinite and the probe orientation around point $P$ may be affected by external forces between the probe and the rectum. For this reason, it may be desirable to control the position of point $T$ instead of point $P$, with the brakes on. This leads to the third controller, called BMC-C:

$$
\tau_{B M C-C}=\tau_{g, b}+\tau_{k, T},
$$

where $\tau_{g, b}$ is defined in Eq. (13) and:

$$
\tau_{k, T}=\mathbf{J}_{v 1, T}^{\mathrm{T}} k\left(\mathbf{x}_{\mathrm{ref}, T}-\mathbf{x}_{T}\right) .
$$

In this last equation, $k$ is a stiffness coefficient, $\mathbf{x}_{T}\left(\operatorname{resp} . \mathbf{x}_{\mathrm{ref}, T}\right)$ is the actual (resp. desired) position of point $T$ in the fixed frame $\mathscr{F}_{0}$, which can straightforwardly be computed from $\mathbf{q}$ though the robot's direct kinematics model and $\mathbf{J}_{v 1, T}$ is the $3 \times 3$ sub-jacobian matrix that maps the velocity of the three active joints to the velocity of point $T$ :

$$
\mathbf{v}_{6 / 0}(T)=\mathbf{J}_{v 1, T}\left(\begin{array}{c}
\dot{\theta}_{1} \\
\dot{\theta}_{2} \\
\dot{\theta}_{3}
\end{array}\right) .
$$

Here again, the spring behavior at point $T$ may lead to position errors in the presence of external disturbances. To cope with this problem, an external integrator for the position of point $T$ is added:

$$
\mathbf{x}_{\mathrm{ref}, T}=\mathbf{x}_{\mathrm{ref}_{0}, T}+\lambda \int_{0}^{t}\left(\mathbf{x}_{\mathrm{ref}_{0}, T}-\mathbf{x}_{T}(\tau)\right) d \tau,
$$

where $\mathbf{x}_{\text {refo }}, T$ is the position of point $T$ recorded when the locked mode is switched. In practice, $\lambda$ is set to $0.4 s^{-1}$. The resulting control scheme is depicted in Fig. 9.

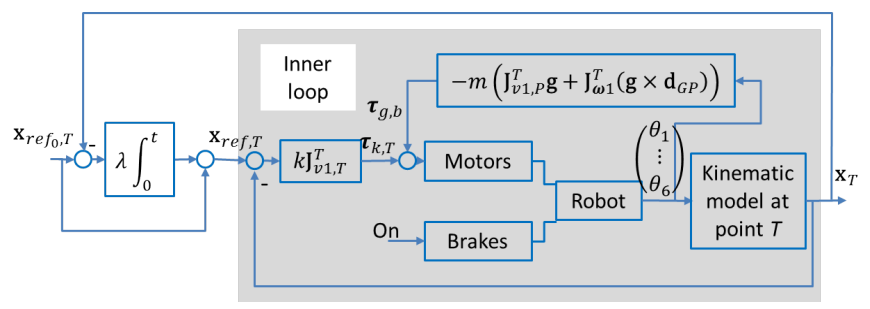

Figure 9. Third control scheme in the locked mode (BMC-C)

Table Table IV summarizes the main differences between the three controllers. Namely, the brakes are not used for controller BMC-A, while only controller BMC-C is exploiting the wrist joint position sensor.

\begin{tabular}{||c|c|c||}
\hline Controller & Wrist brakes & Wrist Position Sensors \\
\hline BMC-A & No & No \\
\hline BMC-A & Yes & No \\
\hline BMC-C & Yes & Yes \\
\hline \multicolumn{3}{|c|}{ Table IV }
\end{tabular}

THE THREE CONTROLLERS PROPOSED FOR THE LOCKED MODE DIFFER IN THE EQUIPMENT THEY REQUIRE FOR THE WRIST JOINTS

\section{B. In vitro experiments}

The ability of the three proposed controllers to lock the probe towards a biopsy target was evaluated during in vitro experiments. During these experiments, a urologist was asked to comanipulate of the probe inserted in a prostate phantom (model 053, manufactured by CIRS). This phantom replicates both the biomechanics of a rectum and a prostate (similar stiffness and friction) and the echogenicity of the prostate. During the experiments, the urologist had to position the probe at twelve different locations, that he selected as the adequate locations for performing a biopsy, according to the sextant scheme (see Fig. 10-up-left).

In order to monitor the adequate positioning of the probe, the urologist was using a Urostation, produced by the company Koelis (La Tronche, France). This system, which is approved for clinical use, is connected to a $3 \mathrm{D}$ ultrasound machine (Sonix RP from Ultrasonix) and includes an algorithm that accurately registers two 3D ultrasound images of a prostate even in the presence of significant deformations. The protocol used for these experiments is similar to the clinical protocol. First, immediately after introducing the probe in the patient's rectum (here: the phantom), the urologist records a reference 3D US image. This image is displayed on a screen interface. Then the urologist moves the probe towards a desired location, 

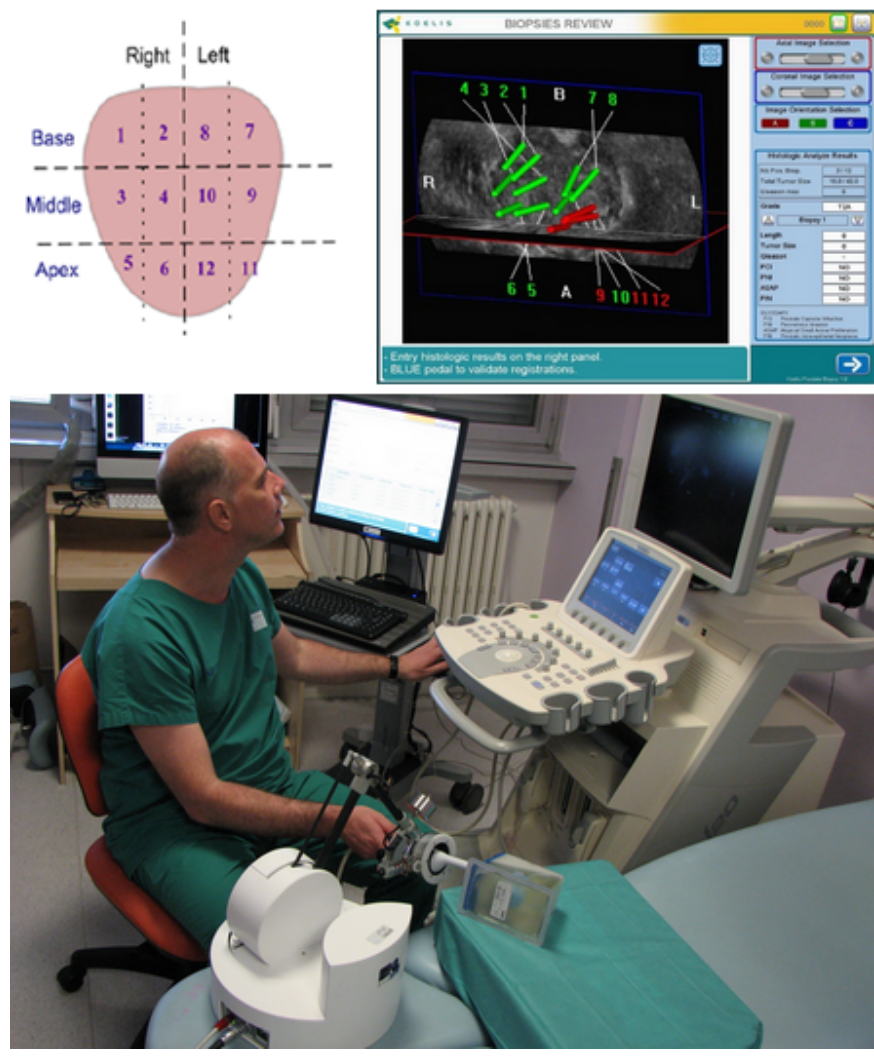

Figure 10. Upper left: the sextant scheme used to position the probe during the in vitro experiments. Upper right: a typical image from Urostation, allowing the urologist to visualize the location of a biopsy in a prostate. Bottom: setup with the phantom and the robot.

following the sextant scheme. To this aim, he uses the real time 2D US image and mental reconstruction of the anatomical geometry. When he thinks he has reached the adequate location, he records a new 3D US image which is registered to the reference 3D image by the Urostation. Knowing the displacement computed by the registration algorithm, the Urostation displays, in the initial reference 3D US image, the expected location of the biopsy needle, represented with a thin cylinder, as illustrated in Fig. 10-up-right. The urologist can then adapt the probe position until he has reached a position he estimates to be satisfactory. At this time, he selects the locked mode for the robot. The joint robot positions are recorded and the three different modes of control for the locked mode were evaluated.

Three controllers summarized in Table IV are tested.

\section{Results}

Figure Fig. 11-left presents the time evolution of the position error of point $P,\left\|\mathbf{x}_{\text {ref }_{0}}-\mathbf{x}\right\|$, recorded during of an example experiment in Mode 1. A large steady state error is observed. It is due to the forces exerted by the prostate phantom on the probe and the low stiffness selected for the robot inner loop due to security constraints. On the contrary, in Fig. 11right, where an example result is presented for Mode 4, it can be seen that the error in the $P$ position converges to zero, as expected from the presence of an integrator. The integrator activity can be visualized by the time evolution of $\left\|\mathbf{x}_{\mathrm{ref}_{0}}-\mathbf{x}_{\mathrm{ref}}\right\|$, which corresponds to the norm of the integrator output.
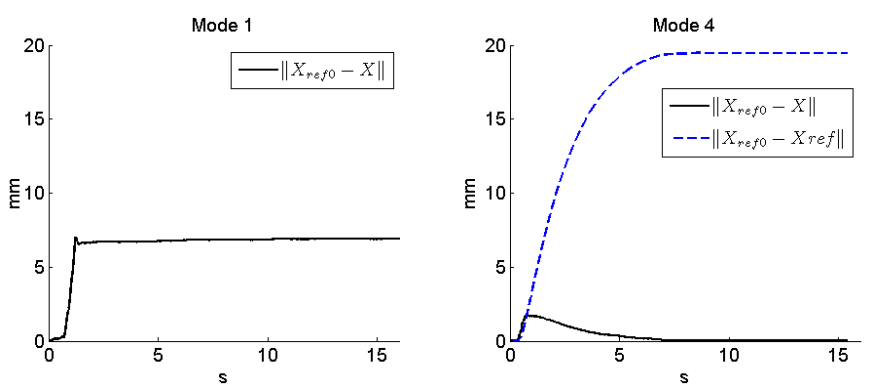

Figure 11. Evolution of the reference and position along the z-axis during one blockade.

Figure Fig. 12 shows the maximal error norm and the steady state error norm for the five experiments in the four modes. In the right column, it can be observed that, as expected, the steady state error is null with the integrator, independently from the fact that the brakes are ON or OFF. The benefit of using brakes lies in the fact that the orientation is maintained constant by the brakes (up to their stiffness, which is high). Therefore, not only the position of $P$ can be kept still but also the orientation. Furthermore, when the brakes are OFF, if can be seen in Fig. 12 that the maximal (transcient) error is significantly increased at point $P$.
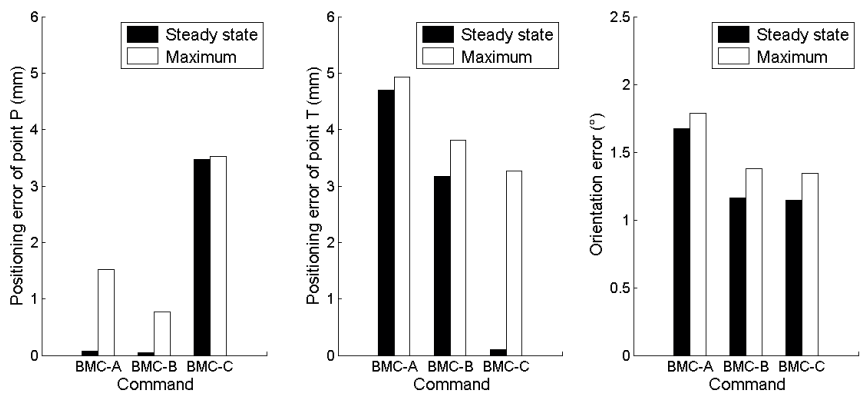

Figure 12. Maximal and steady state errors for each considered point and command law

\section{IN CADAVERO EXPERIMENTS}

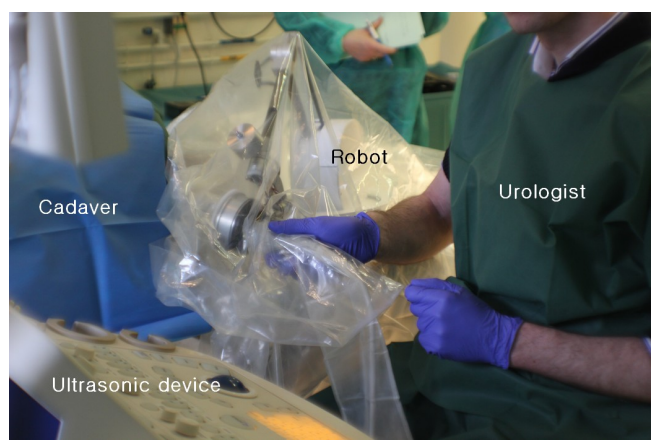

Figure 13. In cadavero experiments

Experiments have been conducted in cadavero at the Surgical School of Assistance Publique - Hopitaux de Paris. Two urologists were comanipulating the system on two fresh cadavers. 
A first aim was to verify the geometry. The two urologists were asked to scan the whole prostate with the probe using the ultrasound image, as they would do during a conventional examination. It appeared that the robot workspace was satisfying, whether the cadaver was in left lateral decubitus or in lithotomy position, lying down with feet in stirrups. No fastidious setup was required for any of the two body positions: the robot was simply positioned on the table or on a stool, without precise pre-positioning. The first try for placing the robot base was satisfactory and convenient to perform all the experiments in both patient positions and for both patients. Moreover, the urologists declared they felt comfortable and not disturbed in their gesture by the robot in free mode.

Experiments similar to the in vitro experiments were then performed to evaluate the three proposed command for Apollo. There were only two differences in the set-up:

- instead of using a phantom, the probe was inserted in a cadaver rectum;

- the prostate couldn't be properly imaged due to the tissue deterioration, thus the Urostation was not used and the urologists based the biopsy targeting on their experience.

Results are presented on Fig. 14.
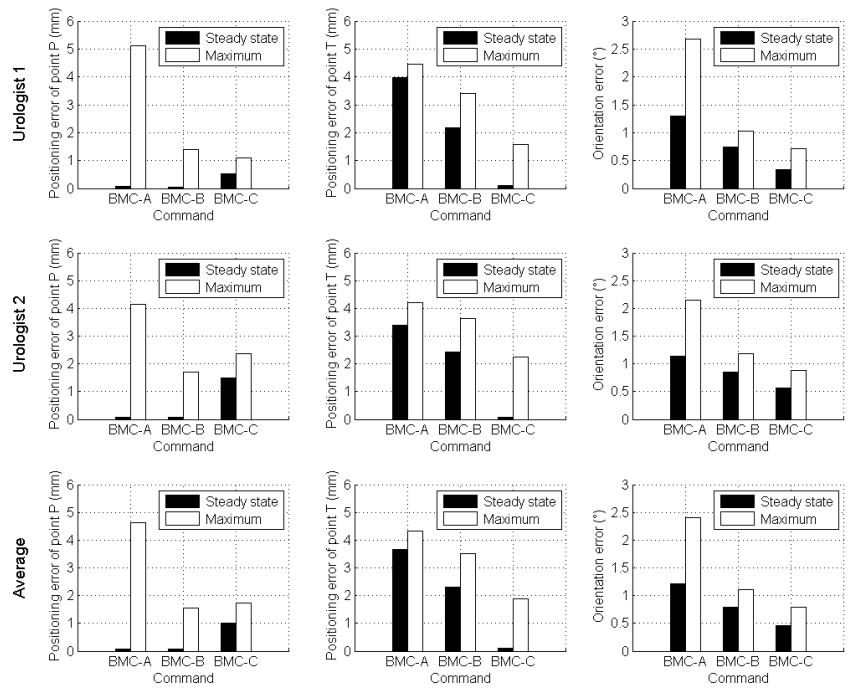

Figure 14. Maximal and steady state errors for each considered point, each command law and each urologist

Those results are consistent with those obtained in vitro for each urologist.

\section{CONCLUSION}

In this paper, we presented the design of a comanipulator for assisting endorectal prostate biopsies. This lightweight system, based on conventional robotic components, possesses 6 degrees of freedom but uses only 3 electric motors and 3 basic brakes. It features a free mode, where its low friction and inertia allows for natural manipulation of the probe and a locked mode, exhibiting both a very low stiffness and a high steady state precision. It can be registered to the prostate by the use of 3D-3D USI registration algorithms.

A step toward clinical application was made thanks to an in cadavero experiments, as the robot appeared to bring significant help in the locked mode while not disturbing the urologist in the free mode. Current developments include a quantified characterization of the manipulation easiness in the free mode and a quantification of the brakes and wrist stiffness. Indeed, in cadavero experiments, coupled with ultrasound imaging of the prostate, emphasized that the orientation is not kept exactly constant by the system. One future development will consider working on expressing the control reference in the ultrasound image frame (visual servoing), in order to improve the effective precision.

\section{REFERENCES}

[1] "American cancer society." [Online]. Available: http://www.cancer.org

[2] P. Stattin, E. Holmberg, J.-E. Johansson, L. Holmberg, J. Adolfsson, J. Hugosson, and on behalf of the National Prostate Cancer Register (NPCR) of Sweden, "Outcomes in localized prostate cancer: National prostate cancer register of sweden follow-up study," Journal of the National Cancer Institute, vol. 102, no. 13, pp. 950-958, 2010.

[3] G. Lu-Yao, P. Albertsen, D. Moore, and et al., "Outcomes of localized prostate cancer following conservative management," JAMA, vol. 302, no. 11 , pp. 1202-1209, 2009.

[4] N. N. Stone, J. Roy, S. Hong, Y.-C. Lo, and R. G. Stock, "Prostate gland motion and deformation caused by needle placement during brachytherapy," Brachytherapy, vol. 1, no. 3, pp. 154 - 160, 2002.

[5] V. Lagerburg, M. A. Moerland, J. J. Lagendijk, and J. J. Battermann, "Measurement of prostate rotation during insertion of needles for brachytherapy," Radiotherapy and Oncology, vol. 77, no. 3, pp. 318 $-323,2005$.

[6] N. Hungr, M. Baumann, J. Long, and J. Troccaz, "A 3-d ultrasound robotic prostate brachytherapy system with prostate motion tracking," IEEE Transactions on Robotics, vol. 28, no. 6, pp. 1382-1397, Dec. 2012.

[7] S. Salcudean, T. Prananta, W. Morris, and I. Spadinger, "A robotic needle guide for prostate brachytherapy," in Robotics and Automation, 2008. ICRA 2008. IEEE International Conference on, May 2008, pp. 29752981.

[8] H. Ho, P. Mohan, E. Lim, D. Li, J. Yuen, W. Ng, W. Lau, and C. Cheng, "Robotic ultrasound-guided prostate intervention device: system description and results from phantom studies," The International Journal of Medical Robotics and Computer Assisted Surgery, vol. 5, no. 1, pp. 51-58, 2009.

[9] G. Fischer, I. Iordachita, C. Csoma, J. Tokuda, S. DiMaio, C. Tempany, N. Hata, and G. Fichtinger, "Mri-compatible pneumatic robot for transperineal prostate needle placement," Mechatronics, IEEE/ASME Transactions on, vol. 13, no. 3, pp. 295-305, June 2008.

[10] A. Patriciu, D. Petrisor, M. Muntener, D. Mazilu, M. Schar, and D. Stoianovici, "Automatic brachytherapy seed placement under mri guidance," Biomedical Engineering, IEEE Transactions on, vol. 54, no. 8, pp. 1499-1506, Aug.2007.

[11] S.-E. Song, N. Cho, G. Fischer, N. Hata, C. Tempany, G. Fichtinger, and I. Iordachita, "Development of a pneumatic robot for mri-guided transperineal prostate biopsy and brachytherapy: New approaches," in Robotics and Automation (ICRA), 2010 IEEE International Conference on, May 2010, pp. 2580-2585.

[12] G. Fichtinger, T. L. DeWeese, A. Patriciu, A. Tanacs, D. Mazilu, J. H. Anderson, K. Masamune, R. H. Taylor, and D. Stoianovici, "System for robotically assisted prostate biopsy and therapy with intraoperative ct guidance," Academic Radiology, vol. 9, no. 1, pp. 60 - 74, 2002.

[13] L. Phee, D. Xiao, J. Yuen, C. F. Chan, H. Ho, H. Choon, C. Christopher, and S. N. Wan, "Ultrasound guided robotic system for transperineal biopsy of the prostate," in Robotics and Automation, 2005. ICRA 2005. Proceedings of the 2005 IEEE International Conference on, April, pp. $1315-1320$.

[14] Z. Wei, M. Ding, D. Downey, and A. Fenster, "3d trus guided robot assisted prostate brachytherapy," in Medical Image Computing and Computer-Assisted Intervention MICCAI 2005, ser. Lecture Notes in Computer Science, J. Duncan and G. Gerig, Eds. Springer Berlin Heidelberg, 2005, vol. 3750, pp. 17-24.

[15] H. Bassan, T. Hayes, R. Patel, and M. Moallem, "A novel manipulator for 3d ultrasound guided percutaneous needle insertion," in Robotics and Automation, 2007 IEEE International Conference on, April 2007, pp. 617-622. 
[16] G. Fichtinger, J. P. Fiene, C. W. Kennedy, G. Kronreif, I. Iordachita, D. Y. Song, E. C. Burdette, and P. Kazanzides, "Robotic assistance for ultrasound-guided prostate brachytherapy," Medical Image Analysis, vol. 12 , no. 5 , pp. $535-545,2008$, special issue on the 10th international conference on medical imaging and computer assisted intervention MICCAI 2007.

[17] T. Podder, W. S. Ng, and Y. Yu, "Multi-channel robotic system for prostate brachytherapy," in Engineering in Medicine and Biology Society, 2007. EMBS 2007. 29th Annual International Conference of the IEEE, Aug.2007, pp. 1233-1236.

[18] M. R. van den Bosch, M. R. Moman, M. van Vulpen, J. J. Battermann, E. Duiveman, L. J. van Schelven, H. de Leeuw, J. J. W. Lagendijk, and M. A. Moerland, "Mri-guided robotic system for transperineal prostate interventions: proof of principle," Physics in Medicine and Biology, vol. 55, no. 5, p. N133, 2010.

[19] C. Schneider, A. Okamura, and G. Fichtinger, "A robotic system for transrectal needle insertion into the prostate with integrated ultrasound," in Robotics and Automation, 2004. Proceedings. ICRA '04. 2004 IEEE International Conference on, vol. 1, April-1 May, pp. 365-370 Vol.1.

[20] A. Krieger, I. Iordachita, P. Guion, A. Singh, A. Kaushal, C. Menard, P. Pinto, K. Camphausen, G. Fichtinger, and L. Whitcomb, "An mricompatible robotic system with hybrid tracking for mri-guided prostate intervention," Biomedical Engineering, IEEE Transactions on, vol. 58, no. 11, pp. 3049-3060, Nov. 2011.

[21] C. Torterotot, P. Mozer, M. Baumann, M. Vitrani, and G. Morel, "Analysis of endorectal probe kinematics during prostate biopsies," in Proc. of the Hamlyn Symposium on Medical Robotics, ISBN: 978-09563776-1-6, 2010, pp. 47-48.

[22] J. Denavit and R. S. Hartenberg, "A kinematic notation for lower-pair mechanisms based on matrices," Trans ASME J. Appl. Mech, vol. 23, pp. 215-221, 1955

[23] "Haption." [Online]. Available: http://www.haption.fr

[24] S. Balasubramanian, A. Melendez-Calderon, and E. Burdet, "A robuste and sensitive metric for quantifying movement smoothness," IEEE Transactions on Biomedical Engineering, vol. 59, no. 8, pp. 2126-2136, 2012. 